\title{
DER GEBRAUCH VON DER UND WELCHER \\ IN RELATIVSÄTZEN.
}

W ustmanns ${ }^{1)}$ aufstellungen taber den gebrauch von der und welcher in relativsätzen haben mich veranlasst, statistische beobachtungen auf grund eines zwar nicht vollständigen, aber immerhin reichen und bunten materiales anzustellen. Eine anzahl von mitgliedern unseres seminars hat mir dazu ihre dienste angeboten. Es wurde aus dem zeitraum von 1750$1850 \mathrm{mehr}$ als ein dutzend von prosawerken ausgewählt und aus ihnen, um jede willkur zu vermeiden, je nach dem format und der beschaffenheit des druckes 1 oder $1 \frac{1}{2}$ bogen, vom anfang des textes an, in untersuchung gezogen. Die folgende tabelle gewährt zunächst eine ubersicht uber die resultate. Sie bezeichnet den titel des werkes, welchem ich die von mir. gebrauchte abkurzung beifuge. Sie gibt dann die anzahl aller nebensätze, der relativsätze mit welcher, der relativsătze mit der und der relativsätze mit was, mozu, warum u. s. w. an.

Aus dieser tabelle ergibt sich zunăchst, dass die gesammtzahl der relativsătze $(1743: 3603)$ nahezu die hälfte aller nebensătze ausmacht und dass durchschnittlich auf je zwei sătze mit der einer mit welcher kommt. Entbehrlich ist welcher als relativum für keinen der in untersuchung gezogenen schriftsteller gewesen. Man findet es bei Lessing in den Literaturbriefen und in der Dramaturgie ungefahr in der durchschnittlichen anzahl, im Laokoon dagegen weit öfter, fast so oft als das demonstrativum gebraucht. Winckelmann kennt fast nur welcher als relativum, das auch in Schillers historischen schriften

1) Allerhand sprachdummheiten, Leipzig, Grunow 1891 s. $144 \mathrm{ff}$. Vgl. meine anzeige in der Wiener zeitung rom 7. bis 9. april 1892 no. $80-82$; im s.-a.: Allerhand sprachgrobheitem, eine höfliche entgegnung von J. Minor, Stuttgart, Cotta'sche buchhamdllung nachfolger 1892. 


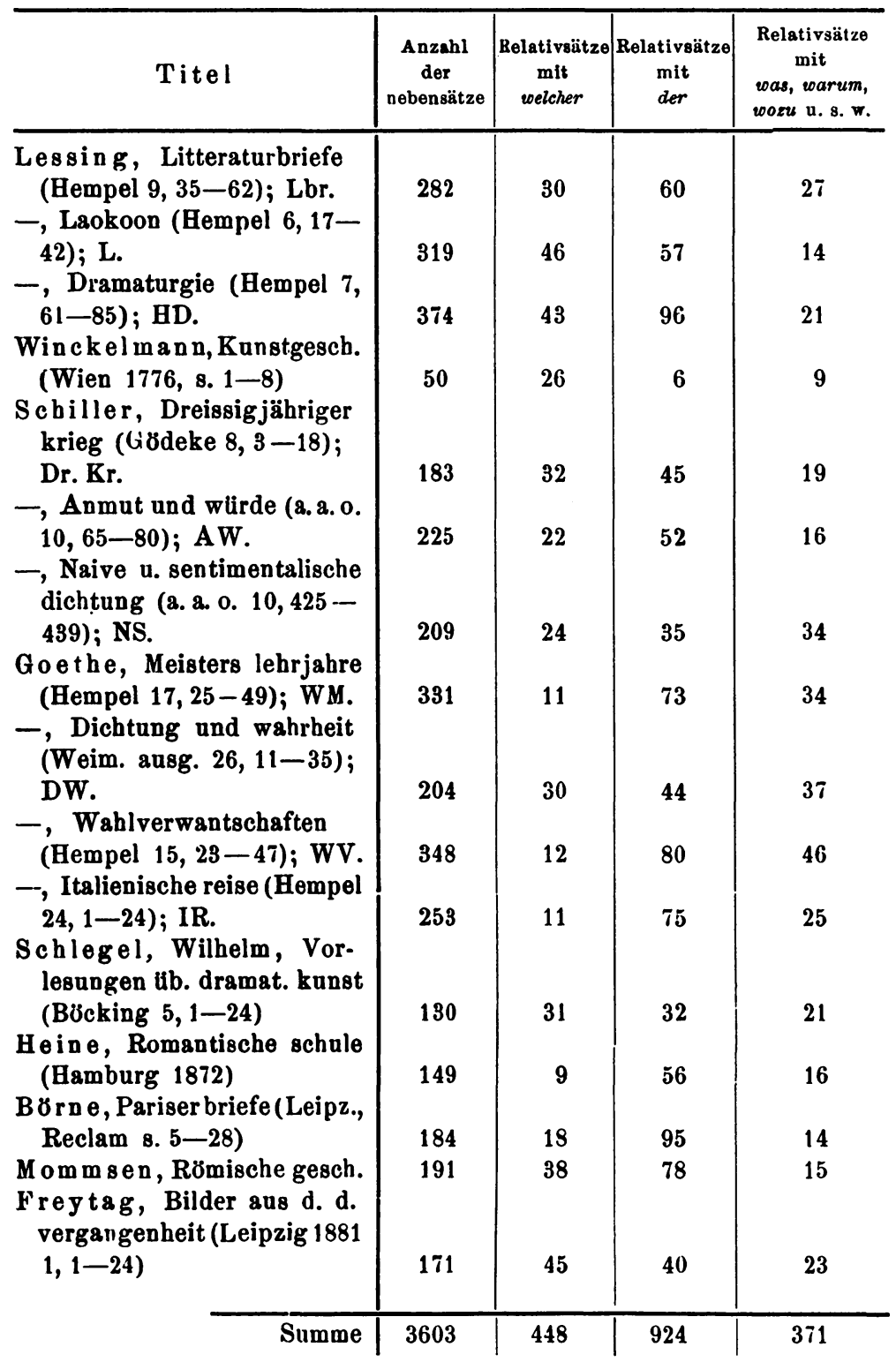

Summe alle r relativsätze $(448+924+371)=1743$. 
dem demonstrativum die wage hält. Aber in der philosophischen abhandlung uber Anmut und wurde sinkt das verhältnis zwischen der und welcher noch unter das normale (52:22) herab und hebt sich in der schlussabhandlung widerum uber das normale (35 : 24) in die böhe. In Goethes Meister, Wahlverwantschaften und in der Italienischen reise kommt ziemlich gleichmässig auf seiten der ein einziges welcher; das verhältnis bleibt ziemlich constant dasselbe $(73: 11,80: 12,75: 11)$. Auffallend dagegen ist der häufige, das normale weit tbersteigende gebrauch von welcher in Dichtung und wahrheit (30 welcher auf 44 der). Heine und Börne zeigen dasselbe verhältnis, das bei Goethe gewöhnlich ist; Heine 9:56, Börne 18:95. Bei den neueren dagegen kommt welcher wider in die höhe. Bei Mommsen ist das verhältnis normal (38:78), aber bei Gustav Freytag stehen 45 welcher gegen $40 \mathrm{der}$. Winckelmann und Freytag sind also die einzigen, bei denen der gebrauch von welcher überwiegt, während sich bei Wilhelm Schlegel der und welcher (32:31) fast genau das gleichgewicht halten.

Interessanter wird die untersuchung, wenn man besondere fälle in betracht zieht. Ich beschränke mich dabei nicht auf die von Wustmann aufgestellten gruppen.

Fur das kennt Lessing fast nur welches. Unter 18 fällen kommen 16 welches und nur 2 das vor: vergnügen, auf welches ich stolz zu sein ursache habe Lbr; das buch, welches den tilel... führt Lbr; das falsche, welches er mit sich führt $\mathrm{L}$; dem leiden gleichmässig, welches der gegenstand äussert $\mathrm{L}$; das vergnügen, welches aus der ähnlichkeit entspringt $\mathrm{L}$; das gesetz der Thebaner, welches ihm ... L; das gesicht des vaters, welches... L ; das drama, welches... L; des mitleidens fähig, welches... L; keines mehr als das, welches... L; das mitleid, welches wir empfinden $\mathrm{L}$; das übel, welches wir fühlen $\mathrm{L}$; ein dunkles gefühl, welches... HD; ein raffinement, welches... $\mathrm{HD}$; das geschmeidige, welches... HD; alles das ausdrückende, welches ihm eigentümlich ist HD. Diesen zahlreichen beispielen stehen bloss die beiden folgenden gegenluber: ein natürliches gefühl, das ... L; jedes wort, das ... HD. Einmal gebraucht Lessing was anstatt das: alles elend, was die menschliche natur treffen kann L. 
Schiller gebraucht welches wohl noch häufiger als das, aber nicht in solchem ubermasse wie Lessing. Alles gute, welches Philipp II. gegen Elisabeth beschloss Dr. Kr; ein wesen, welches selbst ursache... ist $\mathrm{AW}$; ein gemischtes gefühl, welches das (anstatt das das) naive in der denkart ausmacht NS; ein herz..., welches die (anstatt das die) hülfe der kunst... verschmähte NS; ein lächeln, welches doch schwerlich ... zum grunde hat NS; das naive..., welches durch übertragung... auf das vernunftlose entstehet NS; das wolgefallen, welches es ... empfindet NS. Man beachte, dass diese beispiele fast alle aus NS stammen, während die folgenden mit das alle dem Dr. $\mathrm{Kr}$ angehören: ein gemüth empören, das von der ahnung eines besseren schon gewonnen war $\mathrm{Dr} . \mathrm{Kr}$; ein ... interesse, das dem volke näher liegt $\mathrm{Dr} . \mathrm{Kr}$; ein motiv, das ... Dr. $\mathrm{Kr}$; ein vaterland, das ... Dr. Kr; eines stiftes, das ... Dr. Kr. Einmal steht welches auch fur was: als dasjenige ist, welches über jene bestimmung ....AW.

Bei Goethe dagegen findet man nur zweimal welches für das in DW, wo sich uberhaupt vorliebe fur welcher zeigt: ein haus, welches aus zwei... häusern bestand $\mathrm{DW}$; ein zimmer, welches man das gartenzimmer nannte DW. Sonst heisst es immer das: ein packet, das zeitungen von der post geschickt hatte WM; das puppenspiel..., das ich euch ... gebe und das euch ... beibrachte WM; ein porträt, das ... bedeckt war WM; ein bündel, das die alte... betrachtete WM; ein personal, das jene wirkung hervorbrachte WM; das land ... das wir ... durchwandern können WM; ein theater, das er zusammengebaut hatte WM; ein spiel, das die andern sehr unterhielt WM; ein stück, das nicht existierte $\mathrm{WM}$; eines gedichtes, das sich finden muss, in welchem die muse ... sich zankte $\mathrm{WM}$; ein weib, das nicht zugleich liebe einflösste WM; des schicksals, das ihm durch Mariannen die hand reichte WM; das ziel, das er sich vorgesteckt sah WM; ein gewühl, das sich versammelte $\mathrm{DW}$; gesperr, das man bei seite selzen wollte $\mathrm{DW}$; verlangen, das der knabe $z u$ befriedigen suchte $\mathrm{DW}$; ein gesetz, dus man nicht ... dulden wollte DW; etwas vollkommeneres..., das er anderswo gesehen $\mathrm{WV}$; stückwerk, das gefäilt und anregt WV; ein kind, das zum wohl geboren ward WV; etwas vollständiges..., das allen menschen ... ehrfurcht einflösste WV; totliegendes, das jedoch für älter 
gehalten werden muss IR; heidekorn, das sie blende nennen IR; das schloss, das im wasser liegt IR; türkisches korn, das... treibt IR; das einzige haus, das ... IR.

Wilhelm Schlegel bleibt bei welches: das moralische schauspiel, welches man...darbot. Heine wechselt ab: das jüdische volk, welches...galt; das gewand, das vergiftet war. Börne bevorzugt das: echo, das es nachspricht; Paris, das nur 20 meilen entfernt ist; gefrorenes, das ich ...gegessen; volkes, das ... zurückkehrte; volkes, das ... nicht erregt; mein blut, das ... friedlich floss; mein geschick, das mich verurtheilte. Dagegen nur: ein marmorpiedestal, auf welches man die bildsäule... hat stellen wollen; ein künstliches geschwür, welches die (anstatt das die) ...vorsehung ... zuzieht; ein französisch ..., welches die (anstatt das die) kleinstädter nicht verstanden. Mommsen kennt nur das: ein volk, das... lebte; ein wort, das ... bezeichnet; das meer, das die Hellenen...gemacht hat; ein land, das ...thätigkeit anstrengt; das werkzeug, das ... furcht; das widerspiel, das behütet; hellenisches wesen, das opferte. Bei Freytag halten sich das und welches die wage: ein geschlecht, das sich gewöhnt; des hauses, das er sich ... aufgeführt hat; kriegsvolk, das auf rache denkt; ein recht, das den mächtigen scheut; das neue gold, das aus Amerika herübergefahren wird; ein vaterland, um das er sich grämt. Dagegen: barett, welches der hof ... schenkt; ein schicksal, welches viel zeit und geld kostet; ein amt, welches einige kenntnisse fordert; das schicksal, welches sie alle trifft, ein höheres leben, welches ... zur erscheinung kommt; ein leben, welches ... aufbrach; das leben, welches ... dahinströmt.

Nur dort, wo sich das relativum auf einen ganzen satz bezieht und die periode abschliesst, hat sich welches auch bei Goethe und bis in die neueste zeit behauptet, wo man fast ausschliesslich was sagt. Lessing: er liess den harlekin feierlich vom theater vertreiben, welches selbst die grösste harlekinade war, die jemals gespielt worden Lbr. Schiller: würde der verstand richter sein, welches einen widerspruch einschliesst $\mathrm{AW}$; hier konnte... berechnet werden, welches aber nicht miehr stattfindet $\mathrm{AW}$; bekennet sich als unterthan, welches an alle einwohner ergehet. Goethe: David und Goliath anzubringen, welches im regelmässigen drama gar nicht angehen wollte $\mathrm{WM} ;$...., welches denn manchem nachgeborenen mag zu gute gekommen sein $\mathrm{DW} ; . .$. , welches 
denn auch eingetroffen ist DW; fand sich keine gesellschaft..., welches offers geschah $\mathrm{WV}$; glas und bild unversehrt..., welches denn doch ein wunder sei IR; musste ich ihr einen jahrmarkt kaufen, welches ich ihr denn auch versprach IR. Wilhelm Schlegel: welches sie jedoch ... nicht vermöge.

In gewissen casus wird das pronomen welcher nicht mehr gebraucht: man sagt im gen. fem. und pl. nicht welcher sondern deren, im gen. sg. masc. nicht welches sondern dessen. Es verdient aber beachtung, dass sich gerade in diesen casus erweiterte formen von der herausgebildet haben, die das pronomen von dem artikel unterscheiden. Derselbe fall liegt auch im dativ pluralis vor und auch hier wird welchen endlich vor denen den platz räumen müssen. Bei Lessing ist welchen noch weitaus ubberwiegend $(17: 4)$ : an welchen sie theil hatten Lbr (mitten unter formen von der); aus welchen sie gefunden zu haben scheinen Lbr; unter welchen sie diese arbeit von mir verlangen $\mathrm{Lbr}$; von denen verstanden, welchen das beiwort zukam Lbr; leute..., mit welchen... Lbr; zeiten, in welchen er lebte $\mathrm{Lbr}$; die verwünschungen, mit welchen sein schmerz ... das lager erfüllte $\mathrm{L}$; töne der verzweiflung, von welchen auch der dichter das theater... durchhallen liess $\mathrm{L}$; zeiten, aus welchen dieser aufzug besteht $\mathrm{L}$; grade, in welchen sie ... fähig sind $\mathrm{L}$; schönheiten erreicht, von welchen uns ... nie träumen würde $\mathrm{L}$; allen unbequemlichkeiten des lebens, welchen man ausgesetzt ist $\mathrm{L}$; die einzelnen scenen, in welchen Philoktet ... ist L; leidenschaften, mit welchen andere... sympathisieren können $\mathrm{L}$; vorschläge, welchen es zum vorwurf gereichen wird $\mathrm{HD}$; rollen..., in welchen ... HD; bewegungen ..., mit welchen $\mathrm{HD}$. Dagegen bei Lessing nur die folgenden vier fälle mit denen und auch diese erst aus der dramaturgie: mienen, mit denen; regeln ..., nach denen ...; gesten, mit denen ...; schauspieler, denen. Bei Winckelmann natülich immer welchen: in welchen er arbeitete; mit welchen er verkehr hatte etc. Bei Schiller halten sich welchen und denen fast das gleichgewicht: länder, in welchen die katholische religion ... die herrschende war $\mathrm{Dr} . \mathrm{Kr}$; anschauungen, in welchen der reine natursinn sich gefällt $\mathrm{AW}$; bedingungen, unter welchen das schöne sich erzeugt AW. Aber: wohlthätige folgen von denen er begleitet war Dr. $\mathrm{Kr}$; anstrengungen . .., von denen er . . . erschöpft wurde $\mathrm{Dr} . \mathrm{Kr}$; 
klöster. . ., an denen ihre vorfahren mitstiften halfen $\mathrm{Dr} . \mathrm{Kr}$; die ... übel, von denen die aufrichtigkeit . . . bedroht wird NS; handlungen, bei denen wir schlechterdings in zweifel gelassen werden NS. Bei Goethe uberwiegt denen schon weitaus das veraltende welchen: die stücke, in welchen nur mannspersonen auftraten WM; prospekte, mit welchen der vater... geschmückt hatte $\mathrm{DW}$; thierstücke, auf welchen kleine kinder... spielten $\mathrm{DW}$; die briefe lesen, in welchen sie ... beobachtungen mittheilt WV (in einem mitgeteilten briefe); grosse flecken, in welchen sich wieder kleine runde flecken ... zeigen IR. Dagegen: thüren, an denen ich wochenlang vorbeigehen musste $\mathrm{WM}$; die reden Davids, mit denen er Goliath herausforderte WM; arbeiten, bei denen die bedienten im hause... zerbrachen WM; stücke, in denen ich zu gefallen hoffle WM; wolken, in denen seine leidenschaft sie emportrug WM; vorfälle, denen sie ausgesetzt war WM; Eulenspiegeleien, zu denen mich angereizt $\mathrm{DW}$; zimmer, in denen man sie gehalten..., die gänge, auf denen sie gespielt hatte WM; bewachsene hügel, an denen sie sich hinzogen $\mathrm{WV}$; packete, von denen ich ... ein bekenntnis ablegen muss IR; diejenigen dinge, von denen man sich ... unterhalten hätte IR. Sogar bei Schlegel, trotz der vorliebe für welcher, finde ich im dativ pluralis nur denen: lieblingsdichter, in denen so manche züge... nicht zu erkennen waren; die beiden welten, zwischen denen wir uns getheilt fühlen. Auch Mommsen kennt nur denen: zwischen den halbinseln, mit denen ... endigt; die leidenschaflen, auf denen... ruht; die... stürme, auf denen beruht; Italiker, von denen ... sind; revolutionen, von denen ... stattfanden; fundamentalgedanken, auf denen ... beruht. Ja sogar Freytag zieht in diesem casus denen vor; ich finde nur: romane, in denen edle liebende... sich unterhalten; sängern, von denen jeder . . . beansprucht ; vögel, bei denen ... eine einheit darstellt. Dagegen bietet mir Börne nur beispiele für welchen: opfer..., welchen du am freundlichsten lächelst; bürgern, welchen allen hass und wuth im herzen kochte. Bei Börne allein finde ich auich die form welcher als gen. pl.: früchte, wegen welcher Verdum noch heute berühmt ist.

Der gebrauch von welcher wird uberall dort nahegelegt, wo man den zusammenstoss des gleichlautenden artikels oder demonstrativums mit dem relativum aus euphonischen grlinden zu vermeiden sucht. Denn dass unsere scihriftsteller an zusammen- 
stellungen wie der der, die die etc. anstoss nehmen, ist eine tatsache, von der nur Wustmann nichts wissen will. Freilich ist die aufmerksamkeit in dingen dieser art bei den verschiedenen schriftstellern eine verschiedene. Man kann drei fälle unterscheiden:

I. auf ein substantivum mit artikel folgt ein gleichlautendes relativum; also der mann, der oder die frau, die.

In diesem fall trennt nicht bloss ein wort, sondern a uch die satzpause die gleichlautenden pronomen und artikel; sodass das zusammentreffen wenig anstössig erscheint. Gleichwohl kann man deutlich erkennen, dass einzelne schriftsteller auch in diesem fall das zusammentreffen vermeiden, wenn es sich um die femininform die bandelt. Lessing sagt: die lücke, welche Lbr (nicht die lücke, die); die briefe, welche Lbr; die handlung, welche diese (nicht die diese); die bemerkung, welche $\mathrm{L}$; die kunst, welche $\mathrm{L}$; die auslegung, welche $\mathrm{L}$; die religion, welche $\mathrm{HD}$ (bis); die liebenswürdigkeit, welche. Dagegen finde ich nur einmal: die ruhe, die L. Stehen aber zwischen den gleichlautenden pronomen und artikel zwei wörter, dann sagt auch Lessing: die schönen ideen, die er Lbr; die patriotische verachtung, die er $\mathrm{Lbr}$; die schönen linien, die $\mathrm{L}$; die ursache klar, die ich suche $\mathrm{L}$; die religion selbst, die $\mathrm{HD}$; die erste tragödie, die $\mathrm{HD}$. Bei Winckelmann: die bilder, welche...; die hetären, welche ...; die gemeinschaftlichen stoffe, welche ...; dagegen: die werke, die gehen. Bei Schiller völlige gleichgiltigkeit: die freiheit, die nur im olymp zu hause ist $\mathrm{AW}$; die sinnenwelt, die sein einziges object ist $\mathrm{AW}$; die frau, die NS; dagegen: die vernunflideen, welche $\mathrm{AW}$; die veränderungen, welche $\mathrm{AW}$. Bei Goethe finde ich nur zweimal: die von Ochsenstein, welche sahen $\mathrm{DW}$; die kalkalpen, welche ich bisher durchschritten IR. Sonst immer doppeltes die: die leidenschaft, die WM; die wärterin entliess, die $\mathrm{WM}$; die hausapotheke, die $\mathrm{WV}$; die gesellschaft, die den 28. august feiern mochte IR; die milde luft, die ein grosser fluss mitbringt IR; die witterung, die mir so günstig ist IR; die veränderungen, die sich ... zeigen IR; die feuchtigkeit, die in ihr...vertheilt war IR; die wasser, die aus dem berge kommen IR; die unruhe, die hinter mir ist IR; die heuschrecken, die zu schrillen anfangen IR. (Man beachte, dass die mehrzahl der beispiele aus IR stammt.) Umgekehrt geht Wilhelm 
Schlegel wie Lessing dem zusammentreffen zweier die auch hier aus dem weg; er sagt: die theorie, welche; die geistige biegsamkeit, welche; die neu-europäischen völker, welche; die gelehrten, welche; die hauseintheilung, welche; die nordischen eroberer, welche; die naturpoesie, welche; die glänzendste erscheinung, welche; die kleinliche geschäfligkeit, welche; die lücken, welche; die äusserlichen handlungen, welche. Diesen vielen beispielen stehen nur die folgenden drei gegentiber: die bedingungen, die; die kunst, die; die ahndung, die. Auch Heine sagt lieber: die fragmente, welche; die beiden schriften, welche - als: die gelehrten, die. Börne dagegen sagt nur: die wenigen menschen, die; die rechnung, die; die grazie, die; die neuen schlachtfelder, die; die glasgassen, die; die erhöhrung, die; die täuschung, die; die wahrheit, die. Mommsen: die culturgeschichte, die; aspiraten, die; aber auch: die überlieferung, welche. Freytag ist consequenter als alle, selbst als Lessing und Schlegel. Er schreibt nur: die wölfe, welche; die postzeitung, welche; die neue knollenfrucht, welche; die herren, welche; die laune, welche; die bilder, welche.

Anders steht es mit dem weniger helltönenden der. Hier schreibt Lessing zwar auch gern: der erste, welcher... verglich, war ein mann, der ... verspürte $\mathrm{L}$ (nicht: der erste, der ... war ein mann, welcher); der zornige Jupiter, welcher $\mathrm{L}$; der jammer, welcher $\mathrm{L}$; der uneigennützigkeit, mit welcher $\mathrm{HD}$; der theil der action, welcher $\mathrm{HD}$; mit der bewegung, mit welcher HD. Aber es kommt nicht viel seltener vor: der ackersmann, der Lbr; nach der freiheit, zu der Lbr; der dichter, der ihn tadelt $\mathrm{L}$; bei der mässigung, zu der $\mathrm{HD}$; der verfassung, mit der $\mathrm{HD}$; der einfalt, der $\mathrm{HD}$; der sophokleische, der $\mathrm{L}$; der allgemeine, der $\mathrm{HD}$; der langsamste, der $\mathrm{HD}$. Bei Schiller finde ich nur einmal: theil der schönheit, welcher von diesem gebrauch abhängt AW. Sonst immer: der künstler, der sie beabsichtigt $\mathrm{AW}$; der geist, der über die fälle entscheidet $\mathrm{AW}$; der gegenstand, der uns... einflösst NiS; der erste, der NS; der gelehrte, der NS. Bei Goethe ebenso: der vater, der WM; der mond, der $\mathrm{DW}$; der einzige, der $\mathrm{DW}$; der grosse, der WV; der grund, der WV; der erste, der IR. Börne: der rothe farbenstoff der fahne, der in unser motterland hinüberleitete; ein kämpfer in der schlacht, der seinem schmerz kann ausblicken lassen. Man beachte ubrigens, dass in der anzahl der beispiele 
sich das relativum auf eine person bezieht: der einzige, der; und ich glaube beobachtet zu haben, dass von personen häufiger der als welcher gebraucht wird, wenigstens bei einigen schriftstellern.

Noch weniger anstoss erregen den ... den (Lessing: den beifall, den $\mathrm{HD}$; den verlust, den $\mathrm{HD}$. Schiller: den gedanken, den NS. Goethe : den eindruck, den DW) oder das ... (Heine: das theater, das; das ritterthum, das).

II. Ein zweiter fall ist, wenn das demonstrativum unmittelbar vor dem gleichlautenden relativum steht und beide nur durch die satzpause getrennt sind. Hier sagt Lessing. widerum nicht: alle die, die dem staate dienen; sondern: alle die, welche dem staate dienen $\mathrm{Lbr}$; es ist die, welche $\mathrm{HD}$; wenn die, welche $\mathrm{HD}$ (bis). Winckelmann: diejenigen, welche. Goethe: es ist die, welche sich nach Höchst zieht $\mathrm{DW}$; oder er gebraucht diejenigen, die: diejenigen, die an euer gnaden gerichtet sind $\mathrm{WV}$; diejenigen, die... abergläubisch sind $\mathrm{WV}$; diejenige, auf die meine sinne...gerichtet waren WM. Bei Heine: als die, welche der deutsche mönch angezettelt hat. Fälle, in denen die, die vorkommt, stehen mir aus meinem material nicht zu yebote; und doch wären sie unter allen kakophonien noch am leichtesten zu ertragen und zu entschuldigen. Denn es liegt in der absicht des sprechenden, den hinweis möglichst deutlich und stark hervortreten $\mathrm{zu}$ lassen, und das relativum kann sich an das demonstrativum, an dessen stelle es gewissermassen tritt, gar nicht eng genug anschliessen. Der gleichlaut kann also hier in der absicht des redenden gelegen sein. Trotzdem scheint es vermieden zu werden. Bei Goethe findet sich sogar: diejenigen, welche sich gar nicht fuir mich schickten WM; also das von Wustmann verhöhnte diejenigen, welche. Aber was Wustmann dagegen geltend macht, beweist gar nichts. Er findet, dass die zusammenstellung rom volksmund blos parodistisch verwendet wird, und stösst sie deshalb in den abgrund. Aber das beweist doch nur, dass die wendung im mund des dialekt redenden volkes ihre wirkung verfehlt, nicht, dass sie an sich schlecht ist. Wenn alle verse albern wären, die das volk bloss aus dem parodistischen gebrauch kennt, dann stånd es schlimm um unsere literatur. Das urw luchsige nimmt sich im mund des gebildeten oft nicht weniger lächerlich aus, als die schriftsprache im mund des volkes. 
An dem zusammentreffen von $d e r$, der nimmt Lessing auch hier keinen anstoss: auch der, der nicht weiss HD. Am liebsten aber setzt er einmaliges der oder welcher fur der, der oder der, welcher: als der (für der, der) ohne zeit herumirrt HD; beide schaden sich selbst: der (für der, der) zu viel spricht und der zu viel erwartet HD. Auch wo kein misslaut zu vermeiden ist, liebt es Lessing, das relativum unmittelbar an die stelle des unterdrückten demonstrativums zu setzen: denn sie ist zwar eine stumme pause, aber die (eine, die) sich unmittelbar unsern augen verständlich machen will $\mathrm{HD}$; dass der theil der action, welcher dort der feurige war, hier der kältere, und welcher (der, welcher) dort der kältere war, hier der feurige sein muss HD. Uebrigens zieht Lessing nach dem demonstrativum derjenige, das er gern anwendet, und nach dem artikel mit demonstrativer kraft entschieden welcher vor: mit aller der vorsicht, welche L; mit der genauigkeit, mit welcher wir $\mathrm{L}$; in der ordnung niederschreiben, in welcher sie sich entwickelt $\mathrm{L}$; in dem augenblick genommen, in welchem sie ihre kinder ermordet $\mathrm{L} ;$ mit derjenigen wuth nicht zeigen können, welche man vermuthen sollte L; dasjenige gemurmel, durch welches L. Dagegen: ist eben derjenige, den wir kennen; derjenige, den wir schreien hören $\mathrm{L}$; mit dem beifall aufgenommen worden, den $\mathrm{HD}$; in denjenigen rollen, die $\mathrm{HD}$. Man beachte, dass fast alle beispiele aus dem Laokoon stammen. Zusammentreffen des demonstrativums mit dem relativum in verschieden lautenden formen ist ohne anstoss: der, den er getroffen $\mathrm{L}$; von der, die er misskannt L.

III. Im dritten fall stehen das relativum und der artikel gleichlautend neben einander innerhalb des relativsatzes. Es ist der anstössigste fall, weil hier die die, der der, das das durch keine pause getrennt werden.

Trotz Wustmann, der auch an dreifachem die keinen anstoss nimmt, muss nun behauptet werden, dass von den in untersuchung gezogenen schriftstellern alle, ohne ausnahme, dem die die aus dem wege gehen. Es handelt sich nicht allein um einen misslaut, sondern auch um eime der zunge unbequeme aussprache. Denn die die oder gar dïe die die ist ein gestotter, dem man lieber ausweicht.

Lessing sagt obne ausnahme: mach den regeln beurtheilt, 
welche die kunstrichter... abstrahirt haben Lbr; sprüche der dichter, welche die lehrer waren $\mathrm{Lbr} ;$ mit aller vorsicht..., welche die wage gleich erhalten muss $\mathrm{L}$; einer wirkung fähig, welche die nähere aufsicht des gesetzes heischet $\mathrm{L} ;$ grenzen, welche die grazien seiner kunst setzten $\mathrm{L}$; vertiefung, welche die widrigste wirkung von der welt schuf $\mathrm{L}$; unterschiede, durch welche die wirkung...eingeflochten wird $\mathrm{HD}$; regeln, welche die $\mathrm{HD}$; bewegung, welche die $\mathrm{HD}$; ruhe, welche die $\mathrm{HD}$. Ebenso Schiller in weitaus den meisten fällen: welche die freiheit Europas bedrohte $\mathrm{Dr} . \mathrm{Kr}$; welche die regenten hatten $\mathrm{Dr} . \mathrm{Kr}$; welche die nationen absonderte $\mathrm{Dr} . \mathrm{Kr}$; welche die anlage berührte $\mathrm{AW}$; zwecke, welche die natur mit dem menschen beabsichtigt $\mathrm{AW}$; zwecke, welche die natur... mit ihm vorhat $\mathrm{AW} ;$ gunst, welche die vernunft erzeigt $\mathrm{AW}$; ursache, welche die erscheinung bestimmt AW. Ein besonders lehrreicher fall ist dieser aus $\mathrm{Dr} . \mathrm{Kr}$ : Spanien und Italien, aus welchen ländern die österreichische macht ... ihre stärke zog, waren . . Rom mit blinder anhänglichkeit ergeben, welche die... Spanier ausgezeichnet hat. Hier hätte Schiller, der sonst immer in relativsätzen gleicher ordnung zwischen der und welcher abwechselt, sicher im zweiten satze die gesetzt, wenn er nicht an dem zusammentreffen zweier die anstoss genommen hätte. Ebenso bei Goethe in den allermeisten fällen: sonne, gegen welche die fenster gerichtet waren $\mathrm{DW}$; vorsorge, welche die obrigkeit ihren bürgern schuldig ist $\mathrm{DW}$; pforten und thürme, welche die gränze der alten stadt bezeichneten $\mathrm{DW}$; die dritte bank, welche die handwerker einnahmen $\mathrm{DW}$; die verschiedenen bilder, welche die landschaft zeigte $\mathrm{WV} ;$ gegenstände, welche die richtung ... annehmen WV. Ebenso wenn die beiden die nur durch ein kurzes einsilbiges wort getrennt wären: aspekten, welche mir die astrologen sehr hoch anzurechnen wussten $\mathrm{DW}$; jede schönheit, welche durch die neuen wege... sichtbar geworden $\mathrm{WV}$; feigen, welche als die ersten vortrefflich schmeckten IR. Oder wenn zwischen den beiden die nur eine kleine pause wäre: bei den stücken, welche, die meisten lustspiele nicht ausgenommen, in Alexandrinern abgefasst waren DW. Ganz ebenso Schlegel: die kritik ist es, welche die geschichte der künste aufklärt; gärten, welche die kinder anzulegen pflegen; bauart, welche man die gothische baukunst nannte; einige denker, die übrigens die eigenthümlichkeiten ebenso 
bezeichnen. Auch Heine sagt: die einzige kunde, welche die Franzosen erhalten hatten; und Börne: die unverschämtheit der fürstenschmeichler, welche die völker als tiger darstellen. Freytag: eine schlacht, welche die söhne lieferten. Es ist natülich ganz derselbe fall, wenn auf das relativum die ein dies oder diese folgt. Auch da sagt Lessing: die handlung, welche diese; nicht: die diese. Winckelmann: welche dieselben. Schiller: welche dieses $\mathrm{Dr} . \mathrm{Kr}$ (bis); die erste wirkung, durch welche dieser Dr. Kr. Schlegel: die, welche dies annehmen; die lücken, welche diese reden lassen. Börne: in den 3 wagen, welche diesen mittag durchkamen. Und Mommsen: alle anregungen, welche diesen gegensatz hervortreten machten.

Diesen massenhaften beispielen stehen nur die folgenden, ganz vereinzelten fälle gegenuber. Bei Schiller in AW heisst es zweimal: was für eine idee das nur sei, die die vernunft... hineinbringt; anmuth ist die schönheit derjenigen erscheinungen, die die person bestimmt. Bei Go ethe kommt vor: Marianne rief der alten, die die wunderlichen materialien anzupassen beschäftigt war WM. Bei Mommsen: die ältesten aufgaben, die die erde... stellt. Nur ein kleines wort steht zwischen den beiden die in zwei fällen bei Goethe: einige andere, die gegen die strassenecke zu lagen $\mathrm{DW}$; früchte, die erst die rechten sind WV. Börne: die freiheit der franzosen, die ja die freiheit aller völker ist.

In rhythmischen und euphonischen dingen handelt es sich naturlich niemals um schulregeln, auf deren nichtbefolgung eine strafe gesetzt wird. Das ohr des menschen ist gar ein feines und zartes organ, und keineswegs zu allen zeiten gleich empfindlich und scharf. Wenn man aber uberhaupt dort von einem gesetz reden kann, wo sich eine deutliche neigung zu der einen form und eine unzweidentige abneigung vor der andern ausspricht, so darf man als gesetz festhalten, dass unsere besten schriftsteller das zusammentreffen zweier die vermeiden.

Ueber das zusammentreffen des relativums die mit anderen einsilbigen wörtern von gleichem vocal oder gleichem consonantischen anlaut stehen mir nur die folgenden beispiele zur verfügung. die sie Lbr. L. HD. AW. WV widerholt; dagegen welche sie $\mathrm{HD}$. Dr. Kr. AW. WV. IR. Auch wodurch sie für durch die sie Dr. Kr. Die figur die sie sie wird vermieden: 
gegen welche sie sie einzeln nicht bestanden Dr. Kr. Dagegen: die sie sich WH. IR, aber bei Freytag durch welche sie sich. Sogar Winckelmann sagt die sich, auch AW (bis); dagegen welche sich Dr. Kr (dreimal). AW. NS. die der AW (zweimal). WV. Heine; welche der NS. die den $\mathrm{AW}$, welche den Freytag. die durch den NS. die dem AW. Heine viermal; welche dem Börne, Freytag. die das Dr. Kr. WV. IR. die er WV. die du WV. die wir WV, sehr oft. Für die da steht welche da: birnen, welche da meist köstlich sein müssen IR. die ihm $\mathrm{AW}$, aber welche ihn (also den eigentlichen hiatus berorzugend vor dem uneigentlichen) bei Freytag. die in NS (bis). WM; aber welche in NS und bei Freytag. welche individuelle $\mathrm{HD}$. welche immer NS. die ich WM (bis). IR (bis). WV. in die ich mich WM. ich, der ich mich WM. die ich mir WV. die ich dir WV.

Anstatt der der steht welcher der $\mathrm{AW}$. der den: $\mathrm{WV}$ und Freytag (bis). der dem: WM. Heine. der die: Dr. $\mathrm{Kr}$ (dreimal). AW zweimal. WM viermal. IR. Heine. Freytag. Dagegen nur welcher die $\mathrm{DW}$. den die: IR. WM. Heine. der das: AW. IR. WM. WV. Börne; welcher das WV und bei Freytag; welchem das: Freytag. der er: Dr. Kr. Anstatt das die steht welches die NS und zweimal bei Börne; das den bei Freytag. das das und welches das kann ich nicht belegen.

Was endlich den wechsel zwischen der und welcher in relativsätzen betrifft, die demselben satze oder derselben periode angehören, so sind hier die folgenden fälle $\mathrm{zu}$ unterscheiden:

A. Parallele relativaätze, welche von demselben substantiv abhängig sind. Hier habe ich wechsel nur in den folgenden, auffallend seltenen fällen bei Lessing gefunden: thaten, die vor ihren augen geschehen, an welchen sie theil hatten, die zu quellen der unerwartetsten veränderungen wurden Lbr; zeilen, aus welchen dieser aufzug besteht und die... declamiert werden mussten L. Dagegen die folgenden massenhaften beispiele, in denen kein wechsel stattfindet. Mit welcher: dieser rath, welcher aus den bedürfnissen... genommen ist, welcher uns um nichts weiter bringen kann $\mathrm{HD}$; karte, auf welcher das gut ... dargestellt war, und welche der hauptmann sicher zu ergründen wusste $\mathrm{WV}$; die gestaltung, welche schwer ahndet, welche... anerkennt Mommsen; ein geistiger inhalt, bei welchem der an- 
theil . . verschwindet, bei welchem uns . . zur einheit wird Freytag. Mit der: freunde, die ihn an nichts mangel leiden lassen, die sein übel...erleichtern, gegen die er unverhohlen klagen und jammern darf L; worte, deren sinn man einmal gefasst, die man sich einmal ins gedüchtnis eingeprägt hat $\mathrm{HD}$; ein gegenstand, der ...vernichtet und der . . . wiedergeninnt $\mathrm{NS}$; derjenige theil, der nicht bloss... sondern der auch $\mathrm{AW} ;$ dem, der mich liebt und den ich liebe WM; leidenschaft, die ich so oft vorgestellt habe, von der ich einen begriff hatte $\mathrm{WM}$; stellen, die ich auswendig wusste, deren bilder mich umschwebten WM; als eine gottheit ansieht, die nun erst gedeiht, die ihr ehre machen wird $\mathrm{WV}$; der hauptmann, der die überzeugungen anderer nicht gern ... durchkreuzte, den... die erfahrung gelehrt hatte WV; früchte, die erst die rechten kernhaften sind und die sich entwickeln $\mathrm{WV}$; ich kann einen feldchirurgus vorschlagen, der jetzt ... zu haben ist und der mir auch... genüge gethan hat WV; eine witterung, die ich für nichts anderes geben will, die ich aber nicht los werden kann IR; ein mann, den die jesuiten erhiellen und der den verstand verlor IR; der dunkle wald, auf den nie eine ...pflege verwandt ward, der... zum himmel empor wuchs Schlegel; dramatische werke, die von ihren verfassern . . . bestimmt worden sind, die auf ihn keine sonderliche wirkung machen würden; pflegerin meines vaterlandes, die du es wartest, . .., die du hörest

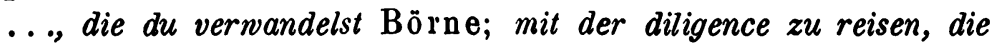
... abgegangen war und die erst ... wiederkehrte; die hand, die unsre ketten zerbrochen, die uns frei gemacht, die uns knechte zu rittern geschlagen; ein kämpfer in der schlacht, der seinen schmerz kann ausblicken lassen und der keine andere schwäche fühlt, als ...; das göttliche widerspiel, das ... behület, das... aber auch geleitet Mommsen; jenes hellenische wesen, das . . opferte, dessen . . lebensideal war, dessen entwicklung... bestand, dessen anschauung ... läugnete, das ...bahn gab, und jenes römische wesen, das ... brannte, das . . forderte, das ... zur pflicht machte, in dem... ein schlechter bürger hiess, wer vermöchte sie auf die einheit zurückzuführen, die sie erzeugte? Mommsen. Endlich noch ein beispiel aus IR, wo die widerholung vermieden und die constructiom aufgegeben ist: indessen war hinter ihm der vorhang zugefallen, den ich lüftete und mich still hielt. 
B. Relativätze von gleicher ordnung, von verschiedenen substantiven abhängig. Hier kommt wechsel im ganzen häufiger vor als beharren; namentlich Lessing und Schiller bieten ihn, weit weniger Goethe. Ich unterscheide, bloss der leichteren ubersicht halber, die folgenden fälle: 1. die relativsätze sind von dem subject und von dem prädicat abhängig; hier stehen mir nur beispiele für den wechsel zu gebot: der erste, welcher die materie verglich, war ein mann, der eine wirkung auf sich verspürte $\mathrm{L}$; diese sichtbare hülle, unter welcher vollkommenheit zur schönheit wird, nur eines von den geringsten mitteln sein kann, durch die er uns zu interessieren weiss $\mathrm{L}$; alles böse, welches Philipp ... beschloss, war rache, die er dafür nahm $\mathrm{Dr} . \mathrm{Kr}$; so war die erste wirkung, durch welche diese allgemeine politische sympathie sich ankündigte, ein dreissigjähriger krieg, der . . ., ein krieg, in welchem . ., der . . Dr. Kr (also auch ein beleg für A mit wechsel). 2. von dem subject und von dem object. Wechsel: eben diese allgemeine staatensympathie, welche den stoss ... dem halben Europa mittheilte, bewacht jetzt den frieden, der diesem krieg ein ende machte $\mathrm{Dr} . \mathrm{Kr}$; die nachdrücklichsten beweggründe, welche von der staatsraison entlehnt sind, lassen den unterthan kalt, der sie selten einsieht und den sie noch seltener interessieren (also auch zu A ohne wechsel). Ohne wechsel: wie weit ist der acteur, der eine stelle nur versteht, von dem entfernt, der sie auch zugleich empfindet $\mathrm{HD}$; lässt er sich in der instruction, die er seinen begleitern... mitgab, zu geständnissen verleiten, die noch bei keinem papste erhört gewesen waren NS. 3. von dem subject und von einer adverbialen bestimmung. Nur beispiele mit wechsel: dass sich der jammer, welcher Agamemnon als vater zukam, durch verzerrungen äussert, die allezeit hässlich sind $\mathrm{L}$; dass uns die ruhe, die wir geniessen, nicht sehr reizend dünken sollte, besonders unter der vorstellung, welche jedes individuum schmeichelt, ... $\mathrm{L}$; er hat das ausdrückende, welches ihm eigenthümlich ist, ohne das beleidigende $z u$ haben, das er in den bildenden künsten... erhält $\mathrm{HD}$; unterscheidet sich von derjenigen, die dem einfluss ..., zum unterschiede von der, welche sich nach freiheit richtet $\mathrm{AW}$. 4. von dem subject und von der apposition; mit wechsel: beim Tasso ist es ein zauberer, ein kerl der weder Christ noch Muhammedaner ist, welcher dem Aladin den rath gibt $\mathrm{HD}$. 5. von 
zwei objecten, ohne wechsel: gegen eine religion, welche das haus Oesterreich beschützt, die anhänglichkeit an eine lehre, welche dieses haus ... zu verfolgen strebte Dr. Kr. Dagegen einem brief der vorsteherin, welcher sich ... verbreitete, war eine nachschrift hinzugefügt ..., die wir beide mittheilen $\mathrm{WV}$. 6. Von parallelen satzgliedern abhängig; hier ist der wechsel selten: in dem grunde, welchen herr Winckelmann... gibt, in der regel, die er herleitet $\mathrm{L}$; voraus ein trupp nationalgarden, welche... schlugen, und dann ein gefolge von bürgern, die ... trugen Börne; auch ein frommer eiferer, der... verdammt, auch der conservative grundherr, welcher für die privilegien eintritt Freytag. Dagegen: die bilder, welche gearbeitet, und andere, welche gegossen wurden Winckelmann; der natur, welche die anlage... gibt, dem glücke, welches das bildungsgeschäft erleichtert AW; die einfachheit überhaupt, welche über die künstelei, und die nalürliche freiheit, welche über steifheit und zwang siegt; das kann nur geschehen bei menschen, die nur dunkel vor sich hin leben, nicht bei solchen, die ... sich ernst benusst sind $\mathrm{WV}$; sie lernt nicht als eine, die erzogen werden soll, sondern als eine, die erziehen will WV; jeder roman, den ich las, jede geschichte, die man mich lehrte WM; im mittelalter fügt man sich mit der resignation, welche uns nöthig ist, oder mit der freudigkeit, welche wir wünschen Freytag; wir empfinden das grössere als einheit, welche ... erscheint, oder als gebilde, welches ... vollendet; todfeind der hirsche..., die seine saaten verwüsten, und nicht neniger feind des schlossherrn, der ihn erschlug; die grossen fenster sehen auf ein dorf, dessen hälften ... aufgebaut sind, und auf eine flur, die erst . . . bestellt wird. 7. Von verschiedenen satzgliedern abhängig; mit wechsel: die bildenden künste insbesondere ausser dem unfehlbaren einflusse, den sie auf den charakter haben, sind einer wirkung fähig, welche die nähere aufsicht des gesetzes heischet L; zwischen mauern, über welche sich traubengeländer sehen lassen, ... andere mauern, die nicht hoch genug sind $\mathrm{IR}$; rings um das ... binnenmeer, das ..., siedelten sich völkerstämme an, welche Mommsen; revolutionen zu führen, von denen... stattfanden, vor der einwanderung, welche führte; die vergleichung der sprachen kann von dem kulturgrade, auf dem ... sie sich befand, ein bild... und die anfänge der geschichte gewähren, welche . . . ist; wir finden den eindruck, den... 
machte, in einer form, welche ... ist. Dagegen: in geistlichen ländern, in welchen die katholische religion die herrschende war, wurde den protestantischen unterthanen (welche es damals schon waren) die freie religionsausübung ausgewirkt $\mathrm{Dr} . \mathrm{Kr}$; zwischen den halbinseln, mit denen... endigt, dehnte sich eine niederung, die ... ist Mommsen.

Es ergibt sich also, dass der wechsel zwischen der und welcher häufiger ist als das beharren, wenn zwei relativsätze von demselben satze, aber von verschiedenen satzteilen abhängig sind.

C. Relativsätze, die derselben periode, aber nicht demselben satze angehören, und entweder von parallelen hauptsätzen oder vom hauptsatz und vom nebensatz abhängig sind. Hier ist das beharren bei demselben relativum häufiger: dass der theil der action, welcher dort der feurige war, hier der kältere, und welcher dort der kältere war, hier der feurige sein muss HD; fand sich keine gesellschaft, welches öfters geschah, so war ... das lesen meist solchen gegenständen gewidmet, welche den wohlstand vermehren $\mathrm{WV}$; die familie ist nicht nur der mittelpunkt, von welchem das einzelne leben... strebt; sie ist auch die schützende mauer, welche dem angehörigen ... sichert Freytag; die frau, die ... verkauft, ist eben so hochachtungswürdig, als der gelehrte ist, der ... verbindet NS; den einen, der ... hinging, liess er liegen, um den andern einzuschlagen, der sich links ... hinaufwand $\mathrm{WV}$; wenn wir jägern gleichen, von denen jeder ... beansprucht, so sind die menschen der vorzeit ... vögeln ähnlich, bei denen ... erst der schwarm eine ... einheit darstellt Freytag; er fühlt sich deutsch im gegensatz zu den Italienern ..., die er hasst, und er sieht. .. auf Frankreich, dessen könig ... verbrennt. Wechsel mit in den folgenden fällen: so wie es tiefsinnige geister... gibt, welche über die religion platterdings wegphilosophiren, ... so gibt es ... schöne geister, die über eben diese religion wegwitzeln Lbr; dort war es diensteifer, der die probe veranlasste, hier ist es die religion, welche gelegenheit ... gibt $\mathrm{HD}$; der Appennin streicht bis zum busen ..., von welchem hervortretend er ... bildet, und nach einer einsattlung, die.... bildet, spaltet er sich Mommsen; so prächtig die krönung .... gewesen war, bei welcher ... der französische gesandte... 
herrliche feste gegeben, so war doch die folge für den guten kaiser desto trauriger, der seine residenz nicht behaupten konnte DW.

D. Relativsätze, von denen der eine dem andern unterge ordn et ist. Hier ist beharren etwas häufiger als wechsel, der gebrauch der einzelnen schriftsteller aber verschieden. Lessing bevorzugt den wechsel, auch Schiller; Goethe wechselt seltener. Gegen Wustmann muss aber behauptet werden, dass im falle des wechsels in den meisten beispielen das zweisilbige welcher den regierenden und das einsilbige der den untergeordneten relativsatz einleitet. Wechsel: welche man den männern, die sich dieser verwaltung unterziehen wollen, nicht anders als beimessen kann $\mathrm{HD}$; welches diejenigen veränderungen hervorbringt, die nicht blos von unserm willen abhangen $\mathrm{HD}$; mit welcher alle stücke, die den acteur ausmachen, gegeben sind $\mathrm{HD}$; aus der beschränktheit unseres zustandes, welche von der bestimmung, die wir einmal erlangt haben, unzertrennlich ist NS; wissenschaftliche erörterungen, welche wenig erfreulich für solche freunde der kunst sind, die nur die hervorbringungen ... geniessen wollen Schlegel; das fabrikwesen beider völker, welches vor mir die berühmtesten deutschen historiker, die sich doch immerfort rühmen ..., übersehen haben Börne; es war ein gemeinsames geistiges leben, welches in tausenden, die zusammenlebten, aufbrach Freytag. Dagegen: die ohnstreitig eine der besten actricen ist, welche das deutsche theater jemals gehabt hat $\mathrm{HD}$; ein ältlicher herr, der, nach seiner ängstlichkeit zu beurteilen, in welche ihn die ... neigung ... versetzte, wohl ein schulmann war Börne; eines hauses, in dessen einem winkel eine kalte krebsscheere stak, welche irgend ein passagier... ausgeh̆höhlt hatte (wo aber dessen im ersten satz gefordert war). Ohne wechsel: ein stärkerer anfall hatte seine gesetzte zeit, nach welchem jedesmal der unglückliche in einen betüubenden schlaf verfiel, in welchem sich seine erschöpfte natur erhohlen musste L; das jüdische volk, welches .... galt und dessen geschichte, welche... hiess, besang Heine; quartblätter, welche ... kunde geben von einer ... schlacht, welche die (statt die die) söhne lieferten Freytag; ein ganzes, an welches eine menge von .... kenntnissen, welche wir in uns tragen, blitzschnell sich anschlossen. Ohne wechsel mit der: ein motiv, das ... einen enthusiasmus ... entflammt, der 
gegen die politische gefahr gerichtet werden kann $\mathrm{Dr} . \mathrm{Kr}$; je grösser die erfurcht war, die ich für die verschlossenen thüren in meinem herzen herumtrug, an denen ich ... vorbeigehn musste und in die ich ... einen blick that, desto schneller war ich, einen augenblick zu benützen, den mich die nachlässigkeit .... treffen liess WM; der vater, der... das gute gedächtniss seines knaben pries, der ... so mancherlei habe behalten können WM; arbeiten, bei denen die bedienten im hause, die arme schneider waren, ... manche nadel zerbrachen $\mathrm{WM} ;$ wir hielten denjenigen für unseren wahrsten freund, der uns bei den brustbildern der sämmtlichen kaiser, die in einer gewissen höhe gemalt waren, etwas von ihren thalen zu erzählen wusste WM; oft schalt... auf die männer, die ... nicht an die kosten denken, die ein erwarteler plan nach sich zieht $\mathrm{WV}$; der staub, der ... den wagen umwirbelt, von dem ich so lange nichts erfahren habe IR; ein werk, das unter allen, die sich nennen lassen, die von der Aeneide verschiedenste gestaltung habe Schlegel; der sterbende centaur, der das gewand, das vergiftet war, überlieferte Heine; die das ganze jahr von dem stoff leben, den ihnen nur ein freies volk verschaffen kann. Bei zahlreicheren beispielen wäre zu beachten, ob bei der unterordnung nicht das zusammentreffen völlig gleichlautender formen (der ... der, die ... die, das ... das) vermieden wird, welches unwillkürlich das gefuhl der gleichstellung hervorrufen könnte.

E. Beiordnung und unterordnung in grösseren perioden. Wechsel ist hier uberwiegend, und mitunter schematisch darstellbar. Ich bezeichne in solchen fällen der mit $\mathbf{A}$ und $\mathbf{a}, \mathrm{je}$ nachdem es im regierenden oder im regierten satz vorkommt; welcher mit $B$ und $b$. Die absicht ..., zu welcher ihre erziehung abzweckte, war, ihre ... bürger zu dem zu bilden, was sie.... nannten, in welchem worte sie ... alle vorgänge begriffen, die einen menschen ... unterscheiden, alle eigenschaften ..., welche den menschen tüchtig machen. $Z u$ dieser absicht, welche $\mathrm{Lbr}[\mathrm{BB}(\mathrm{a}+\mathrm{b})]$; unter den trauerspielen, die auf uns gekommen sind, zwei stücke sich finden, in welchen der körperliche schmerz nicht der kleinste theil des unglücks ist, das den leidenden helden trifft $\mathrm{L}[\mathrm{ABa}]$; so war die erste wirkung, durch welche diese allgemeine sympathie sich ankündigte, ein dreissigjähriger krieg, der ...., ein krieg, in welchem ..., der... 
$\operatorname{DrKr}[\mathrm{A}(\mathrm{A}+\mathrm{B}+\mathrm{A})]$; gründe genug, die ihn hinderten sich für eine partei zu erklären, welche das ansehen des papstes zernichtete, die ihn aufforderten ... $[\mathrm{Ab}+\mathrm{A}]$; eine urkunde dieser ... gesetze, verbunden mit natürlicher aufrichtigkeit, welche ... verbessert (nicht rohheit, welche sich ... hinwegsetzt) erzeugen eine naivheit, ... welche darin besteht, dinge, die man entweder gar nicht oder nur künstlich bezeichnen darf, mit ihrem rechten namen zu benennen $\mathrm{NS}[\mathrm{BB}+\mathrm{Ba}]$; der mensch ist ein wesen, welches selbst ursache ist, welches sich nach gründen, die es aus sich selbst nimmt, verändern kann $\mathrm{AW}[\mathrm{B}+\mathrm{Ba}]$; ein junger mann, den ich ... fragte, zeigte mir ein haus, das man des teufels nennt, welches der ... zerstörer ... erbaut IR $[\mathrm{A}(\mathrm{A}+\mathrm{B})] ;$ eines gedichtes, das sich unter meinen papieren finden muss, in welchem die muse der dichtkunst und eine andere frauengestalt, in der ich das gewerbe personifiziert hatte, sich zankten WM $[\mathrm{A}+\mathrm{Ba}]$; gäste, die mir gefielen, die meinem herzen stoff gaben, der ausreichle bis zum einschlafen Börne $[\mathrm{A}+\mathrm{Aa}]$; voraus ein trupp nationalgarden, welche .. trommeln schlugen, und dann ein gefolge von bürgern, die... standarten trugen, welche mit schwarzen flören behängt und deren inschriften mit immortellen behängt waren Börne [BA $(\mathrm{b}+\mathrm{a})]$; die gäste sind theils stegreifjunker, welche ... von reiterstücken erzählen, die sie ...verübt, theils jüngeres geschlecht, das sich gewöhnt ... zu beugen; diese tragen das barett, welches der hof $\ldots$ schenkt Freytag $[(\mathrm{Ba}+\mathrm{A}) \mathrm{B}]$. In den meisten fällen findet also wechsel von welcher und der ohne rucksicht auf die gliederung des satzes, auf uber- und unterordnung statt.

Endlich sei auch noch der fall in betracht gezogen, wenn das relativum zwischen zwei sätzen eingeklemmt ist. Beispiele mit der: leute, die, weil sie sich selbst am besten kennen, bei jedem guten unternehmen nichts als nebenabsichten erblicken $\mathrm{HD}$; leuten zu statten kommt, die ich weiss nicht welchen schauder empfinden $\mathrm{HD}$; ein allgemeiner satz, der, als solcher, einen grad ... erlangt $\mathrm{HD}$; ein umstand, der, obgleich zufällig scheinend, die patriotisch gesinnten mit besorgnis erfüllt $\mathrm{DW}$. In fast doppelt so vielen fällen dagegen welcher: eine doppelte anmerkung machen, welche, recht behalten, ... bewahren kann $\mathrm{HD}$; durch alle schlingen des falschen geschmackes, in welchen, wenn es klug ist sie ... zu vermeiden, das nichtgenie ... ver- 
strickt wird NS; bei den slücken, welche, die (anstatt die, die) meisten lustspiele nicht ausgenommen, in Alexandrinern abgefasst waren DW; das romantische schauspiel, welches man, genau genommen, ... nennen kann; die äusserlichen handlungen, welche sonst, um den zuhörern klar zu werden, der erzählung bedürfen; ein ort, welcher dem, wo die handlung vorgefallen sein soll, ähnlich ist; von denjenigen dichtern, welche, unbekümmert um die klassischen vorbilder, ihren eigenen weg gegangen sind. Es ist klar, dass das zweisilbige welcher besser geeignet ist zwei sätze aus einander zu halten als das einsilbige der; während der sprechende über das einsilbige der leichter hinwegkommt. Wo also die gliederung scharf hervortreten und sinnfällig gemacht werden soll, wird man zu dem zweisilbigen relativum greifen: welche, | recht behalten, | uns bewahren kann. Dagegen in dem satze: 'die ich weiss nicht welchen schauder emp/inden' kann der sprechende tuber den zwischensatz 'ich weiss nicht welchen' rasch hinwegeilen und hier empfiehlt sich die kürzere form des relativums.

Schliesslich mache ich darauf aufmerksam, dass das relativum welcher, nach Wustmann ein papiernes wort, in den Grimmischen märchen keineswegs selten ist. Ich führe, ohne anspruch auf vollständigkeit, die folgenden belegstellen an: 1, 5 keine anderen als jene, welchen er das leben gerettet hat; 149 sie blies die kohlen an, bei welchen sie ... etwas kochte; 219 dass er in dasselbe wirthshaus gerieth, in welchem seinem bruder das tischchen vertauscht war; 261 machte das fenster auf, unter welchem die räuber standen; 290 zu dem baum kommen, auf welchem das mädchen sass; 382 das tuch, in welches der name der königstochter gestickt war; 397 der jüngste, welcher der däumling hiess; 447 zu dem loch, durch welches sie gekommen waren; 2, 21 und flocht ihre locken still fort, welches der alte könig beobachtete; 119 vor einem grossen wald, durch welchen der weg nach der königsstadt ging; 131 die schuhe, in welchen das schneiderlein ...tanzte; 149 ein loch, durch welches sie kriechen mussten; 343 der schneider, welcher dem kampf zugesehen hatte; $318 \mathrm{f}$. er liess mich in einem gefüngnisse liegen, in welchem mich ein tiefer schlaf befiel. Unter den bildern, welche an meiner seele vorübergingen ... Verwandelte sich in lebendige menschen, in welchen das fräulein ihre diener... erkannte; 363 eine thür, an 
welche die (statt die die) räuber kiopften und die sich alsbald öfnete; 371 ein schiffchen, in welchem seine treulosen gefährten sassen... Ist es blosser zufall, dass auf das relativum in den meisten beispielen der artikel folgt oder weichen die Grimmischen märchen zusammenstellungen wie dem das, der das, das der aus? Interessant aber ist, dass gerade die in den anmerkungen aus dem volksmund mitgeteilten und nicht uberarbeiteten märchen das relativum welcher oft auch mehrmals in einem satz gebrauchen: 3,41 ff. zu dem brunnen wieder, durch welchen er herabgekommen war; nicht ein einzig haar ausziehen, welches sie endlich ... zusammenbrachte; eine grüne wiese, auf welcher ihnen ein sănger begegnete; 76 dann erschrak sie so, dass sie den schlüssel, welchen sie in der hand hielt, in ein becken mit blut fallen liess, welches nicht gut wieder abzuwaschen war.

Eingehende specialuntersuchungen mulssten zeigen, in wie weit sich meine aufstellungen durch ein vollständiges material belegen oder widerlegen liessen. Wie Drobisch in seinen statistischen untersuchungen über den hexameter, habe ich die ersten bogen als vertreter des ganzen werkes gelten lassen, obwol eine abweichung von ihrem gebrauch in der fortsetzung sehr leicht möglich ist. Ein abschliessendes urteil würde nur ein vollständiges material gewähren und die untersuchung durfte sich dann nicht auf einzelne werke eines dichters beschränken. Es wird nicht als ubertlussige arbeit erscheinen, mit einem kleineren, aber bunten material proben angestellt und auf die erscheinungen vorläufig aufmerksam gemacht zu haben, die zunächst in betrachtung gezogen werden mulssten.

WIEN.

J. MINOR. 